\title{
Capítulo III - Tratamento
}

\section{Objetivos do tratamento da asma}

Os objetivos principais do tratamento da asma são:

- Controlar sintomas

- Prevenir limitação crônica ao fluxo aéreo

- Permitir atividades normais - trabalho, escola e lazer

- Manter função pulmonar normal ou a melhor possível

- Evitar crises, idas à emergência e hospitalizações

- Reduzir a necessidade do uso de broncodilatador para alívio

- Minimizar efeitos adversos da medicação

- Prevenir a morte

\section{Medicamentos mais utilizados}

Os medicamentos para asma podem ser divididos em duas categorias, conforme o objetivo da sua utilização: 1 ) fármacos para melhora dos sintomas agudos ( $\beta_{2}$-agonistas com rápido início de ação, brometo de ipratrópio e aminofilina); 2) fármacos para manutenção, usados para prevenir os sintomas (corticosteróides inalatórios e sistêmicos, cromonas, antagonistas de leucotrienos, $\beta_{2}$-agonistas de longa duração e teofilina de liberação lenta).

\subsection{Broncodilatadores $\beta_{2}$-Agonistas}

Os broncodilatadores mais usados na prática clínica são os $\beta_{2}$-agonistas, que podem ser classificados em de curta ação, como o salbutamol, a terbutalina e o fenoterol, cujo efeito broncodilatador dura aproximadamente quatro a seis horas, ou de longa ação, como o salmeterol e o formoterol, com efeito de até 12 horas.

A maioria dos efeitos dos $\beta_{2}$-agonistas é mediada pela ativação da adenilciclase e da produção intracelular de AMP cíclico ${ }^{(1)}$. O $\beta$-receptor é constituído de sete domínios inseridos na membrana celular, dispostos em círculo. Os $\beta_{2}$-agonistas de curta duração estimulam domínios alcançados externamente, enquanto os de longa duração devem penetrar na membrana para estimular lateralmente o receptor, daí seu início retardado de ação(2). 0 formoterol exibe os dois mecanismos de ação, sendo de longa duração e de início rápido de ação.

O $\beta$-receptor é acoplado à proteína $G$ e sua ligação leva a subunidade da proteína $\mathrm{G}$ a estimular a adenilciclase e a produção de AMP cíclico(2). Este ativa a proteína quinase $A$, que produz a maioria dos efeitos celulares do $\beta$-receptor.

Os $\beta_{2}$-agonistas são parcialmente seletivos para os receptores $\beta 2$, concentrando o seu efeito sobre a musculatura brônquica e poupando o sistema cardiovascular de paraefeitos indesejáveis.
Os broncodilatadores $\beta_{2}$-agonistas de curta ação são as medicações de escolha para o alívio dos sintomas da asma, enquanto os $\beta_{2}$-agonistas de longa ação são fármacos que, associados à terapia de manutenção com corticosteróides inalatórios, atuam para o melhor controle dos sinto$\operatorname{mas}^{(3)}$.

0 emprego de $\beta_{2}$-agonistas de curta duração como tratamento isolado de manutenção não é recomendado; o uso freqüente (mais de duas vezes por semana) indica necessidade de tratamento antiinflamatório(4).

Quando administrados por via inalatória, esses medicamentos resultam em menos taquicardia e tremor. Raramente desencadeiam arritmias graves. As opções disponíveis para uso inalatório são: solução para nebulização, aerossol dosimetrado e inaladores de pó. Em doses elevadas, podem contribuir para hipopotassemia. Efeitos centrais são incomuns e incluem cefaléia, ansiedade, sedação, fadiga, náuseas e vômitos. Pode haver hipoxemia, em geral discreta, por piora da relação ventilação/perfusão. Em tratamento intensivo, os $\beta_{2}$-agonistas têm sido empregados por via endovenosa ou subcutânea.

Os $\beta_{2}$ de longa duração têm efeito por até 12 horas. Duas preparações são disponíveis: salmeterol e formoterol. 0 início de ação do formoterol é rápido, semelhante ao dos $\beta_{2}$ de curta duração (um minuto), ao passo que o salmeterol tem início de ação em 20 minutos, com pico de efeito entre duas e três horas, aproximadamente.

Esses medicamentos não têm ação antiinflamatória, não devendo ser usados isoladamente para o tratamento da asma $^{(5-9)}$.

Em caso de crise, um $\beta_{2}$ de ação rápida deve ser preferido, não se reduzindo seu efeito se tiver havido uso prévio de $\beta_{2}$ de ação prolongada (locais distintos de estimulação do $\beta$-receptor) ${ }^{(10)}$. Entretanto, há evidências de que o formoterol induza melhor controle da asma que os $\beta_{2}$ de curta ação, podendo ser também utilizado como droga de resgate ${ }^{(11)}$.

À semelhança dos agentes de curta duração, pode-se demonstrar tolerância em nível laboratorial, com perda do efeito broncoprotetor, com o uso contínuo destes agentes, porém a importância clínica não foi estabelecida.

Os efeitos adversos dos $\beta_{2}$ de ação prolongada são semelhantes aos dos $\beta_{2}$ de curta duração e incluem tremor, taquicardia e hipocalemia ${ }^{(12,13)}$.

Vários estudos demonstraram que a adição de $\beta_{2}$-agonistas de ação prolongada ao corticosteróide inalado leva a melhor controle da asma do que a duplicação da dose do corticosteróide ${ }^{(14-17)}$. 


\subsection{Anticolinérgicos}

O brometo de ipratrópio é o único anticolinérgico disponível para uso inalatório no Brasil. Seu efeito broncodilatador se deve à redução do tônus colinérgico intrínseco das vias aéreas. Possui início lento de ação, com efeito máximo entre 30 minutos e uma hora após a administração(18). Sua ação broncodilatadora é inferior à dos $\beta_{2}$-agonistas e sua utilização é limitada no manejo da asma a longo prazo. $\mathrm{Na}$ asma aguda grave, tem efeito adicional aos $\beta_{2}$-agonistas com comprovada relação custo-efetividade $^{(19)}$. 0 brometo de ipratrópio é o tratamento de escoIha para broncoespasmo induzido por betabloqueadores.

\subsection{Xantinas}

A teofilina e a aminofilina são broncodilatadores de baixa potência e elevado risco de efeitos colaterais ${ }^{(20)}$. Todavia, além de broncodilatadoras, as xantinas parecem ter alguma ação antiinflamatória, equiparável à dose baixa de beclometasona inalatória ou equivalente ${ }^{(21)}$.

A aminofilina é uma opção secundária de broncodilatador para alívio imediato dos sintomas da asma ${ }^{(22)}$. Seu uso como medicação de alívio deve restringir-se a pacientes hospitalizados, de preferência em infusão contínua.

As teofilinas de liberação lenta podem ser administradas como fármacos de controle, para evitar exacerbações ${ }^{(21,23)}$. São superiores às de ação curta por determinarem menor oscilação dos níveis séricos, melhorarem a adesão e oferecerem melhor proteção contra a asma noturna. Entretanto, sua eficácia para o controle de sintomas noturnos é inferior à obtida com uso dos $\beta_{2}$-agonistas de longa duração, por via inalatória(24).

A margem terapêutica das xantinas é muito estreita, isto é, a dose tóxica é próxima da dose terapêutica. Por outro lado, seus níveis séricos, devido ao seu metabolismo hepático, podem ser marcadamente afetados por diversos fatores, incluindo idade, dieta, doenças e interações com outras drogas, tudo contribuindo para a complexidade no uso seguro destas medicações ${ }^{(20)}$. Entre as drogas utilizadas para tratamento da asma, as xantinas têm o maior potencial para toxicidade grave. Sintomas gastrointestinais podem ser intoleráveis para alguns pacientes, mesmo nas doses terapêuticas usuais. No início do tratamento os efeitos colaterais podem ser reduzidos por elevação gradual das doses. Náuseas, diarréia, vômitos, cefaléia, irritabilidade e insônia são comuns quando a concentração sérica excede $20 \mathrm{mcg} / \mathrm{ml}$, e convulsões, encefalopatia tóxica, hipertermia, dano cerebral e morte podem ocorrer em concentrações séricas maiores ${ }^{(20)}$. $\mathrm{Hi}$ perglicemia, hipocalemia, hipotensão e arritmias cardíacas podem também ocorrer, especialmente após superdosagem aguda. Pacientes idosos e lactentes têm maior risco de toxicidade.
A bamifilina é uma xantina com menor incidência de efeitos colaterais (exceto reações de pele), mas faltam estudos comparando sua eficácia em relação à teofilina.

\subsection{Corticosteróides}

São os fármacos de escolha no tratamento de manutenção dos pacientes com asma persistente. Após penetrar na célula, o corticosteróide une-se a um receptor, sendo então transportado ao núcleo, onde se liga a seqüências do DNA, resultando em indução ou supressão de diversos genes envolvidos na produção de citocinas, moléculas de adesão e receptores relevantes no processo da inflamação( ${ }^{(25)}$. Estudos de biópsias brônquicas em pacientes com asma demonstraram que os corticosteróides inalados reduzem o número e a ativação das células inflamatórias e a HR das vias aéreas. Enquanto a redução dos sintomas é rápida, melhora significativa da inflamação e da função pulmonar ocorrem em dias ou semanas, e a modificação da HR, ao longo de vários meses.

\subsubsection{Corticosteróides inalatórios}

Os corticosteróides inalatórios são os fármacos que oferecem melhor relação custo/risco/benefício para o controle da asma persistente. Sua utilização tem sido associada à redução da mortalidade e das hospitalizações por asma ${ }^{(3,4,26)}$.

A terapia inalatória com corticosteróides na asma só foi possível com a introdução de agentes que reuniram máxima potência tópica e mínima biodisponibilidade sistêmica. Estas características foram alcançadas com os agentes lipossolúveis de alta afinidade ao receptor e rápida inativação na primeira passagem pelo fígado, após absorção sistêmica. Logo após o uso do corticosteróide inalado, parte da droga depositada na orofaringe é deglutida e absorvida. A biodisponibilidade sistêmica total é a soma das frações absorvidas do pulmão, trato digestivo e mucosa oral(25).

Existem, entretanto, diferenças de potência clínica quando as doses nominais das diversas preparações são comparadas: flunisolida $\cong$ triamcinolona $<$ beclometasona $<$ budesonida < fluticasona (Q uadro 1)(3). Budesonida e fluticasona têm melhor índice terapêutico que os demais.

\begin{tabular}{|c|c|}
\hline \multicolumn{2}{|c|}{$\begin{array}{l}\text { QUADRO } 1 \\
\text { Comparação de potência tópica } \\
\text { entre corticosteróides }\end{array}$} \\
\hline Droga & Potência tópica \\
\hline Beclometasona & 600 \\
\hline Budesonida & 980 \\
\hline Flunisolida & 330 \\
\hline Fluticasona & 1.200 \\
\hline Triamcinolona & 330 \\
\hline
\end{tabular}


Os dispositivos para administração podem afetar a potência terapêutica dos corticosteróides inalatórios. A budesonida por sistema Turbuhaler ${ }^{\circledR}$ tem deposição duplicada em relação ao aerossol dosimetrado, aumentando a eficácia clínica(27).

Consideram-se doses baixas/médias de corticosteróides inalatórios as inferiores a $800 \mathrm{mcg}$ de beclometasona/dia em adultos e $400 \mathrm{mcg} / \mathrm{dia}$ em crianças, e, doses altas, acima desses valores ${ }^{(3)}$.

Os corticosteróides inalatórios são usualmente utilizados duas vezes ao dia. Em pacientes graves, ou durante exacerbações, a freqüência poderá ser elevada para três ou quatro vezes ao dia ${ }^{(28,29)}$.

\subsubsection{Corticosteróides Sistêmicos}

Prednisona ou prednisolona são os corticosteróides mais utilizados e, por terem meia-vida intermediária, induzem menos efeitos colaterais.

Os corticosteróides orais para controle podem ser necessários em alguns pacientes com asma persistente grave, não estabilizados com outros medicamentos. Deve-se buscar a dose mínima suficiente para controlar cada paciente e administrá-la de uma só vez. Uso em dias alternados pode reduzir os efeitos adversos.

Os corticosteróides sistêmicos são essenciais nas exacerbações quando não há resposta satisfatória aos broncodilatadores, devem ser usados precocemente, e preferencialmente por via oral. A dose, a duração do seu uso e a via de administração dependem da gravidade da crise, da história prévia de exacerbações graves, do uso prévio de corticosteróide e da dose do corticosteróide inalatório em uso corrente. Em pacientes com exacerbações leves, em uso de doses baixas de corticosteróide inalatório, a dose pode ser apenas aumentada. Em crises graves ou em pacientes já em uso de doses elevadas de corticosteróide inalatório, corticosteróide oral ou parenteral deve ser empregado.

\subsubsection{Efeitos colaterais locais}

Candidíase oral é infreqüente quando os corticosteróides inalatórios são administrados com espaçadores e quando os pacientes lavam a boca imediatamente após o uso. Rouquidão é comum e reversível e deve-se à miopatia dos músculos da laringe, não sendo reduzida por espaçadores. Tosse e irritação na garganta, algumas vezes acompanhadas de broncoconstrição, podem ocorrer com os aerossóis dosimetrados devido aos propelentes ou detergentes associados. Este fenômeno não costuma ocorrer com os inaladores de pó.

\subsubsection{Efeitos colaterais Sistêmicos}

A supressão do eixo hipotálamo-hipófise-adrenal pelos corticosteróides depende da dose, duração, forma de uso e formulação empregada. Não existe efeito supressor significativo com doses de beclometasona ou equivalente $\leq$ $1.500 \mathrm{mcg} / \mathrm{dia}$ em adultos e $\leq 400 \mathrm{mcg} / \mathrm{dia}$ em crianças, se cursos de corticosteróide oral não forem prescritos com freqüência ${ }^{(30)}$. 0 teste mais sensível para sua detecção é a dosagem do cortisol na urina de 24 horas ou o teste de estimulação com o ACTH. A medida do cortisol sérico tem baixa sensibilidade (50\%). Em pacientes em uso crônico de corticosteróide oral que iniciam a substituição pelo inalatório, a suspensão das doses orais deve ser cuidadosa, pois a recuperação adrenal pode ser lenta (até três anos). Indivíduos em uso contínuo de corticosteróide oral que sofrem trauma maior (cirurgia ou doença grave) podem necessitar de doses suplementares dessa medicação.

Osteoporose é observada em $30 \%$ a $50 \%$ dos indivíduos em uso crônico de corticosteróide sistêmico, variando com a dose e o tempo de utilização. O controle da asma com corticosteróides inalatórios com conseqüente retirada dos orais é atualmente a estratégia mais efetiva para prevenir perda óssea significativa. A osteoporose pode ser minimizada com atividade física regular, exposição controlada ao sol, alimentação rica em cálcio e uso oral de bifosfonatos ou reposição de estrógenos em muIheres na pós-menopausa(31).

Estudos populacionais sugerem que pode haver risco de catarata e glaucoma em idosos em uso de altas doses de corticosteróide inalatório por tempo prolongado(32).

Adelgaçamento da pele e equimoses são dependentes da dose e do tempo de uso, tendo maior prevalência em idosos.

Estudos de longa duração demonstraram que os corticosteróides inalatórios podem desacelerar o crescimento de crianças, mas não teriam efeito sobre a estatura final(33,34).

Miopatia aguda pode ocorrer com altas doses de corticosteróides no tratamento da crise, particularmente se forem associados a bloqueadores neuromusculares. Miopatia crônica é rara, sendo mais comum com o emprego dos corticosteróides fluorados.

Diabete melito é mais freqüente em pacientes em uso de corticosteróides sistêmicos.

Não há aumento no risco de infecções em pacientes recebendo corticosteróides inalatórios ou prednisona em doses abaixo de $10 \mathrm{mg} / \mathrm{dia}$.

\subsection{CROMONAS}

Embora consideradas antiinflamatórias, a redução da hiper-responsividade brônquica observada com a utilização das cromonas é modesta(35). 0 nedocromil e o cromoglicato bloqueiam os canais de cloro da membrana celular dos mastócitos, eosinófilos, nervos e células epiteliais(36). Os canais de cloro são usualmente fechados, mas abrem-se quando existe ativação celular, com entrada de cálcio e degranulação dos mastócitos. As cromonas res- 
tauram os canais para a posição fechada, aumentando o limiar para sua ativação.

Ambas as drogas são seguras e têm raros efeitos colaterais. 0 nedocromil não está mais disponível em nosso meio e o cromoglicato tem como maior desvantagem a necessidade de ser administrado quatro vezes ao dia.

\subsection{Antagonistas dos leucotrienos}

Os antileucotrienos bloqueiam a síntese ou as interações com os receptores dos leucotrienos. Os agentes em uso clínico no Brasil são montelucaste e zafirlucaste (antagonistas dos receptores).

Seu efeito broncodilatador é modesto, lento, e inferior ao obtido com os $\beta_{2}$-agonistas, embora possa ser aditivo a estes.

Os antileucotrienos têm efeito antiinflamatório e, com uso prolongado, reduzem a hiper-responsividade das vias aéreas ${ }^{(37,38)}$. Têm também efeito protetor na broncoconstrição induzida pelo exercício. Sua indicação deve ser considerada em pacientes com asma persistente ou sintomas induzidos por exercício(38,39).

Alguns estudos demonstraram que, em pacientes com asma de maior gravidade, os antileucotrienos podem permitir a redução do corticosteróide inalado, sem perda do controle da doença, particularmente em asmáticos com sensibilidade à aspirina ${ }^{(40)}$.

Embora alguns casos de vasculite de Churg-Strauss tenham sido descritos com o emprego de antileucotrienos em pacientes que interromperam o uso de corticosteróides orais, não há muitas referências de outros efeitos adversos importantes ${ }^{(41)}$.

\section{Tratamento de manutenção}

A introdução precoce de tratamento antiinflamatório pode resultar em melhor preservação da função pulmonar a longo prazo, prevenindo o remodelamento das vias aéreas. Por outro lado, a asma leve pode permanecer inalterada por muitos anos.

Alguns pacientes desenvolvem obstrução irreversível após muitos anos de atividade da doença. A percentagem é pequena e, provavelmente, os com formas mais graves têm risco maior. Igualmente, faltam dados definitivos demonstrando que a terapia antiinflamatória proteja completamente contra lesões irreversíveis das vias aéreas. 0 tratamento atual é dirigido para controlar os sintomas e prevenir crises.

\subsection{Princípios do tratamento de manutenção}

- Todos os pacientes com asma devem receber orientação sobre sua doença e noções de como eliminar ou controlar fatores desencadeantes, especialmente domiciliares e ocupacionais.

- A educação dos pacientes e familiares pode ser feita em bases individuais ou em grupos.
- As diferenças entre tratamento broncodilatador sintomático e tratamento de manutenção regular devem ser enfatizadas.

- O paciente deve entender a doença e seu tratamento; em casos graves, um medidor de pico de fluxo deve ser utilizado para melhor auto-controle.

- Todos os pacientes com asma persistente moderada ou grave devem ter um plano de ação escrito para uso em caso de exacerbações.

- A terapia deve focalizar de forma especial a redução da inflamação, evitando-se o contato com alérgenos e enfatizando 0 uso precoce de agentes antiinflamatórios na asma persistente.

- Deve-se iniciar o tratamento de acordo com a classificação da gravidade da asma. Havendo dúvida, inicia-se por uma etapa mais elevada.

- Uma vez obtido o controle por período aproximado de três a seis meses, podem-se reduzir as doses das medicações, mantendo-se o paciente monitorizado.

- Tentativas para reduzir a dose dos corticosteróides inalatórios são apropriadas se o paciente alcançou estabilidade, indicada por sintomas esporádicos, normalização da função pulmonar e uso ocasional de $\beta_{2}$-agonistas para alívio. Por outro lado, a cessação completa dos corticosteróides inalatórios provavelmente resultará em deterioração clínica, em tempo variável, estando relacionada ao grau de $\mathrm{HR}^{(42)}$.

- Sempre que o controle esperado não for obtido, antes de quaisquer mudanças deve-se considerar:

- a adesão do paciente ao tratamento;

- a técnica de uso dos dispositivos inalatórios;

- a presença de fatores desencadeantes e/ou agravantes, como sinusite crônica, refluxo gastroesofágico, exposição a novos alérgenos, distúrbios psicossociais e outros.

- Curso breve de corticosteróide oral (cinco a 10 dias) pode ser necessário em qualquer etapa em caso de exacerbação (prednisona 1 a $2 \mathrm{mg} / \mathrm{kg} / \mathrm{dia}$ ou $40 \mathrm{mg} / \mathrm{dia}$ ou equivalente), sem haver necessidade de redução gradual(43).

\subsection{Etapas do tratamento de manutenção}

\section{I - Asma intermitente}

Utilizar $\beta_{2}$ de curta duração por via inalatória para alívio dos sintomas.

\section{II - Asma persistente leve}

- Utilizar $\beta_{2}$ de curta duração por via inalatória para alívio dos sintomas.

- Iniciar terapia antiinflamatória de manutenção. A primeira escolha é corticosteróide inalatório, por exemplo, beclometasona $(400$ a $800 \mathrm{mcg} / \mathrm{dia}$ em adultos e 200 a $400 \mathrm{mcg} / \mathrm{dia}$ em crianças) ou outro corticosteróide inalatório em doses equivalentes. Alternativas: 
- antileucotrienos para quem prefere a via oral ou

- cromoglicato dissódico, especialmente para crianças.

\section{III - Asma persistente moderada}

- Utilizar $\beta_{2}$ de curta duração por via inalatória para alívio dos sintomas.

- Manter as mesmas doses de corticosteróide inalatório da etapa II associando a $\beta_{2}$ de longa duração ou duplicar a dose de corticosteróide inalatório.

- Quando os sintomas persistirem com os tratamentos anteriores, utilizar doses elevadas de corticosteróide inalatório, associado a $\beta_{2}$ de longa duração com ou sem antileucotrienos e/ou teofilina.

\section{IV - Asma persistente grave}

- Utilizar corticosteróide por via oral na menor dose necessária para controle dos sintomas, mantendo as medicações da etapa anterior.
No Quadro 2 estão indicados os esquemas de tratamento em cada etapa.

\subsection{Dispositivos para administraÇÃo de MEdicamen- TOS POR VIA INALATÓRIA}

A deposição pulmonar dos medicamentos depende dos tipos de dispositivos inalatórios. A escolha do dispositivo mais adequado depende da análise de vários aspectos tais como(27,44-49):

1. A satisfação e a adesão do paciente ao tratamento pode ser melhorada se for respeitada, dentro do possível, sua escolha pessoal por determinado dispositivo.

2. A escolha deve ser baseada na otimização do custo/benefício considerando fatores ligados ao paciente, à droga e aos dispositivos disponíveis.

3. A maioria dos pacientes, quando bem orientados, consegue usar eficientemente os aerossóis dosimetrados.

\begin{tabular}{|c|c|c|c|c|}
\hline \multicolumn{5}{|c|}{$\begin{array}{c}\text { QUADRO } 2 \\
\text { Tratamento da asma de acordo com a gravidade }\end{array}$} \\
\hline \multirow[t]{3}{*}{ Gravidade } & \multirow[t]{3}{*}{ Etapa } & \multicolumn{3}{|c|}{$\begin{array}{c}\text { Tratamento } \\
\text { Em todas as etapas - educação e controle } \\
\text { de fatores desencadeantes ou agravantes }\end{array}$} \\
\hline & & \multirow[t]{2}{*}{ Alívio } & \multicolumn{2}{|c|}{ Manutenção } \\
\hline & & & 1ae escolha & Altemativas \\
\hline Intermitente & I & $\begin{array}{l}\beta_{2} \text { curta } \\
\text { duração }\end{array}$ & & \\
\hline $\begin{array}{l}\text { Persistente } \\
\text { leve }\end{array}$ & II & $\begin{array}{l}\beta_{2} \text { curta } \\
\text { duração }\end{array}$ & $\mathrm{Cl}$ dose baixa & $\begin{array}{c}\text { Antileucotrieno } \\
\text { Cromoglicato - crianças }\end{array}$ \\
\hline $\begin{array}{l}\text { Persistente } \\
\text { moderada }\end{array}$ & III & $\begin{array}{l}\beta_{2} \text { curta } \\
\text { duração }\end{array}$ & $\begin{array}{c}\mathrm{Cl} \text { baixa/média dose }+\beta_{2} \\
\text { longa duração } \\
\mathrm{Cl} \text { alta dose }+\beta_{2} \text { longa duração }\end{array}$ & $\begin{array}{c}\mathrm{Cl} \text { alta dose } \\
\mathrm{Cl} \text { alta dose }+\beta_{2} \text { longa duração } \\
+ \text { antileucotrieno ou teofilina } \\
\text { de liberação lenta }\end{array}$ \\
\hline $\begin{array}{l}\text { Persistente } \\
\text { grave }\end{array}$ & IV & $\begin{array}{l}\beta_{2} \text { curta } \\
\text { duração }\end{array}$ & $\begin{aligned} \mathrm{Cl} \text { dose alta } & +\beta_{2} \text { longa duração } \\
& +\mathrm{CO}\end{aligned}$ & $\begin{array}{l}\mathrm{Cl} \text { dose alta }+\beta_{2} \text { longa duração } \\
+\mathrm{CO}+\text { antileucotrienos ou } \\
\text { teofilina de liberação lenta }\end{array}$ \\
\hline
\end{tabular}


4. Em pacientes com dificuldade de uso dos aerossóis dosimetrados, inclusive quando acoplados a espaçadores, a melhor alternativa são os inaladores de pó.

5. Nebulizadores de jato são reservados para crises de asma grave, crianças com menos de três anos e idosos debilitados ou com dificuldade cognitiva que não conseguem usar corretamente ou não se adaptam aos aerossóis dosimetrados acoplados ou não aos espaçadores ou inaladores de pó.
6. Sempre que possível, usar apenas um tipo de dispositivo para facilitar o aprendizado da técnica e melhorar a adesão ao tratamento.

7. A escolha deve levar em consideração a apresentação dos medicamentos/dispositivos disponíveis e facilidade de transporte (os pacientes podem ter dificuldade de carregar espaçadores de grande volume).

8. Para reduzir os efeitos adversos, pacientes que necessitam de altas doses de corticosteróides inalatórios

\begin{tabular}{|c|c|}
\hline & \\
\hline Aerossol dosimetrado - (spray) & Inaladores de pó (IP) \\
\hline $\begin{array}{l}\text { - Retirar a tampa } \\
\text { - Agitar o dispositivo } \\
\text { - Posicionar a saída do bocal verticalmente } 4 \text { a } 5 \mathrm{~cm} \\
\text { da boca } \\
\text { - Manter a boca aberta } \\
\text { - Expirar normalmente } \\
\text { - Acionar no início da inspiração lenta e profunda } \\
\text { - Fazer pausa pós-inspiratória de no mínimo } 10 \\
\text { - segundos } \\
\text { Repetir após } 15 \text { a } 30 \text { segundos para novo aciona- } \\
\text { mento }\end{array}$ & \multirow{3}{*}{$\begin{array}{l}\text { - Preparo da dose: } \\
\text { Aerolizer: retirar a tampa do IP e colocar uma } \\
\text { cápsula, em seguida perfurá-la, comprimindo as } \\
\text { garras laterais. } \\
\text { Turbuhaler: retirar a tampa, manter o IP na verti- } \\
\text { cal, girar a base colorida no sentido anti-horário } \\
\text { e depois no sentido horário até escutar um "click". } \\
\text { Diskus: abrir o IP rodando o disco no sentido anti- } \\
\text { horário, em seguida puxar sua alavanca para trás } \\
\text { até escutar um "click". } \\
\text { Pulvinal: retirar a tampa, manter o IP na vertical, } \\
\text { apertar o botão marrom com uma mão, girar o IP } \\
\text { no sentido anti-horário com a outra mão (apare- } \\
\text { cerá marca vermelha), em seguida, soltar o botão } \\
\text { marrom e girar o IP no sentido horário até escutar } \\
\text { um "click" (aparecerá a marca verde). } \\
\text { - Expirar normalmente e colocar o dispositivo na } \\
\text { boca } \\
\text { - Inspirar o mais rápido e profundo possível (flu- } \\
\text { xo mínimo de } 30 \text { L/min) } \\
\text { - Fazer pausa pós-inspiratória de } 10 \text { segundos } \\
\text { - IP de dose única, fazer nova inspiração, mais } \\
\text { profunda que a anterior, se restar pó na cápsu- } \\
\text { la. }\end{array}$} \\
\hline Aerossol dosimetrado (AD) acoplado a espaçador & \\
\hline \multirow{3}{*}{$\begin{array}{l}\text { - Retirar a tampa do AD e agitá-lo } \\
\text { - Acoplar o AD ao espaçador e posicionar a saída } \\
\text { - Expirar normalmente } \\
\text { - Colocar o bocal do espaçador na boca ou a más- } \\
\text { cara sobre a boca e o nariz, se criança muito pe- } \\
\text { quena ou idoso com grande dificuldade de uso } \\
\text { - Acionar o AD e logo em seguida iniciar inspira- } \\
\text { ção lenta e profunda pela boca ou fazer quatro a } \\
\text { cinco respirações em volume corrente - idosos, } \\
\text { crianças, paciente em crise } \\
\text { - Fazer pausa pós-inspiratória de no mínimo } 10 \text { se- } \\
\text { gundos } \\
\text { - Repetir todas as etapas anteriores para cada acio- } \\
\text { namento do AD }\end{array}$} & \\
\hline & Nebulizadores de jato \\
\hline & $\begin{array}{l}\text { Reservados para crises graves e para pacientes que } \\
\text { não se adaptam aos dispositivos anteriores } \\
\text { - Diluir o medicamento em } 3 \text { a } 5 \mathrm{ml} \text { de soro fisioló- } \\
\text { gico } \\
\text { - Adaptar a máscara à face (boca e nariz) } \\
\text { - Utilizar ar comprimido ou oxigênio a } 6 \mathrm{~L} / \mathrm{min} \text { ou } \\
\text { compressor elétrico } \\
\text { - Respirar em volume corrente }\end{array}$ \\
\hline
\end{tabular}


devem acoplar espaçador ao aerossol dosimetrado e lavar a boca após o uso de inaladores de pó.

9. Independentemente do grau de dificuldade de aprendizado da técnica, é essencial conferir e reorientar periodicamente o uso adequado de cada dispositivo.

Nos Quadros 3 e 4 estão relacionadas as técnicas de utilização e as vantagens e desvantagens dos dispositivos.

\subsection{IMUNOTERAPIA}

- A imunoterapia deve ser administrada por especialista treinado no manejo de reações anafiláticas graves e deve ser indicada apenas na asma atópica, demonstrada por IgE específica aumentada e testes cutâneos de hipersensibilidade imediata positivos.

\section{QUADRO 4}

Vantagens e desvantagens dos principais dispositivos inalatórios

\section{Vantagens}

Desvantagens

\section{Aerossol dosimetrado - AD (spray)}

- compacto e portátil

- múltiplas doses

- menor custo do que IP

- disponíveis para a maioria das drogas

- beclometasona HFA - maior deposição pulmonar (ainda não disponível)
- técnica de uso difícil (requer coordenação disparo/inspiração)

- requer propelentes - CFC lesa camada ozônio

- efeito freon - cessação da inspiração pelo gás frio na orofaringe

- alta deposição na orofaringe

- dificuldade para saber se está vazio

- temores: "viciar e lesar coração"

\section{Aerossol dosimetrado + espaçador}

- facilita o uso do AD

- reduz a deposição na orofaringe (10x)

- pode aumentar a deposição pulmonar

- possibilita o uso de AD nas crises, em volume corrente
- grande volume dificulta transporte

- volume de acordo com a idade

- suscetível aos efeitos de carga estática

- manutenção e limpeza periódicas

- custo de aquisição

- deposição pulmonar variável (depende do conjunto dispositivo, droga, técnica)

\section{Inalador de pó - IP}

- técnica de uso mais simples

- (aerossol gerado e disparado pela inspiração)

- compacto e portátil

- sem propelentes

- múltiplas doses com alguns IP

- marcador de dose em alguns IP

\section{Nebulizador de jato - N J}

- uso em volume corrente

- uso em obstrução grave

- uso de mistura de medicamentos

- pode ser usado em qualquer idade

- baixa deposição na orofaringe

- alta percentagem aerossóis de 1 a $5 \mu$
- dificuldade de preparo da dose

- custo mais elevado

- alguns podem ser afetados pela umidade

- requer esforço inspiratório mínimo (30L/m)

- má percepção de que a dose foi inalada em alguns IP

- custo de compra do compressor ou do oxigênio

- requer fonte de energia, muito ruído

- débito variável, marcas ineficientes

- baixa adesão por dificuldade de transporte ou demora na inalação

- risco de uso de doses excessivas

- requer manutenção e limpeza

- disponíveis apenas para dois corticosteróides 
- Pacientes com sensibilização múltipla (vários alérgenos) podem não se beneficiar do tratamento imunoterápico. É importante verificar cuidadosamente se a profilaxia ambiental está sendo realizada e demonstrar outros desencadeantes que possam estar envolvidos.

- A imunoterapia é mais efetiva em crianças e adolescentes do que em adultos. Não deve ser indicada para indivíduos com menos de cinco ou com mais de 60 anos.

- Por razões de segurança, o paciente deverá estar fora de crise no momento da aplicação da injeção (efeitos colaterais graves têm sido observados em indivíduos com asma grave em crise aguda).

- Para pacientes em terapia farmacológica, antes de administrar a injeção, é aconselhável verificar se $0 \mathrm{VEF}_{1}$ está acima de $70 \%$ do previsto.

- Asmáticos leves ou muito graves devem ser excluídos.

- Não está indicada para os pacientes que respondem bem à profilaxia ambiental e ao tratamento farmacológico.

- Contra-indicá-la para pacientes com outras doenças imunológicas ou auto-imunes associadas, nos que recebem drogas betabloqueadoras e para aqueles sem adesão ao tratamento.

- Avaliar a relação custo/benefício/adesão ao tratamento frente ao poder aquisitivo do paciente.

- Avaliar a confiabilidade dos extratos disponíveis.

\subsection{Acompanhamento}

Pacientes com asma persistente devem ser avaliados regularmente. Nos classificados como moderados e graves, especial atenção deve ser dada ao crescimento e à função pulmonar (PFE, espirometria) a cada consulta, com avaliação oftalmológica e densitometria óssea anualmente. Nos pacientes graves é aconselhável a utilização do PFE matinal antes do uso de broncodilatadores.

A espirometria deve ser realizada para avaliação do controle da asma e sempre que houver mudança no esquema terapêutico.

\subsection{EnCAMINHAMENTO aO ESPECIALISTA}

Os pacientes devem ser encaminhados ao especialista nas seguintes situações:

- dúvida sobre o diagnóstico da doença (ex.: asma vs. DPOC, tosse persistente, dispnéia de causa inaparente)

- provável asma ocupacional

- asma de difícil controle - asma instável, sintomas contínuos apesar de altas doses de corticosteróide inalatório ou necessidade de uso de corticosteróide sistêmico para controle

- piora da asma na gravidez

- adesão pobre ao tratamento e problemas psicossociais
- alta hospitalar recente

- entidades clínicas complicando a asma (ex.: sinusite crônica, refluxo gastroesofágico persistente grave).

\section{Tratamento da cRISE}

As crises de asma devem ser classificadas segundo sua gravidade (Quadro 5) e o tratamento instituído imediatamente(50-52).

\subsection{IdentificAÇÃO do ASMÁtICO dE RISCO}

A causa da morte por asma é asfixia na quase totalidade dos casos. Tratamento excessivo, como causa de óbito, é raro. Por isso, é preciso identificar os seguintes aspectos que indicam maior risco para os pacientes(53):

- três ou mais visitas à emergência ou duas ou mais hospitalizações por asma nos últimos 12 meses

- uso freqüente de corticosteróide sistêmico

- crise grave prévia, necessitando intubação

- uso de dois ou mais tubos de aerossol dosimetrado de broncodilatador/mês

- problemas psicossociais (ex.: depressão)

- co-morbidades - doença cardiovascular ou psiquiátrica

- asma lábil, com marcadas variações de função pulmonar (> 30\% do PFE ou do VEF $F_{1}$ previstos)

- má percepção do grau de obstrução

No Quadro 6 estão mostradas as indicações para a realização de exames complementares.

\subsection{Tratamento medicamentoso}

- O tratamento deve ser baseado no quadro clínico e, quando possível, na avaliação objetiva da limitação ao fluxo aéreo pela espirometria ou PFE. Em crianças com menos de seis anos de idade, utilizam-se os critérios de Wood-Downes(54,55).

- Doses adequadas e repetidas de $\beta_{2}$-agonista por via inalatória a cada 10-30 minutos na primeira hora constituem a primeira medida(22).

- 0 tratamento inicial pode envolver o uso de oxigênio, doses altas repetidas de $\beta_{2}$-inalatório (associado ou não a anticolinérgico) e corticosteróide sistêmico. Todos os pacientes com $\mathrm{SaO}_{2} \leq 95 \%$ devem receber oxigênio(54).

- 0 efeito do $\beta_{2}$-agonista por nebulizador de jato é o mesmo que o obtido por aerossol dosimetrado acoplado a espaçador. A administração por aerossol dosimetrado com espaçador valvulado de grande volume é eficaz mesmo em casos de crise muito grave $\left(\mathrm{VEF}_{1}<30 \%\right)$ e pode resultar em reversão mais rápida da obstrução(56).

- Em adultos, o efeito máximo é obtido, em geral, com 8-16 jatos do aerossol dosimetrado, devendo ser fornecidos 4-5 jatos a cada 15 minutos; em crises graves podem ser fornecidos até oito jatos a cada 10-15 minutos ou um 


\begin{tabular}{|c|c|c|c|}
\hline \multicolumn{4}{|c|}{$\begin{array}{c}\text { Q UADRO } 5 \\
\text { Classificação da intensidade da crise de asma em adultos e crianças }\end{array}$} \\
\hline Achado* & Muito grave & Grave & Moderada/leve \\
\hline Gerais & Cianose, sudorese, exaustão & Sem alterações & Sem alterações \\
\hline Estado mental & Agitação, confusão, sonolência & Normal & Normal \\
\hline Dispnéia & Grave & Moderada & Ausente/leve \\
\hline Fala & $\begin{array}{c}\text { Frases curtas/monossilábicas } \\
\text { Lactente: maior dificuldade } \\
\text { alimentar }\end{array}$ & $\begin{array}{l}\text { Frases } \\
\text { incompletas/parciais } \\
\text { Lactente: choro curto, } \\
\text { dificuldade alimentar }\end{array}$ & Frases completas \\
\hline Musculatura acessória & $\begin{array}{l}\text { Retrações acentuadas ou em } \\
\text { declínio (exaustão) }\end{array}$ & $\begin{array}{c}\text { Retrações subcostais e/ou } \\
\text { esternoclidomastóideas } \\
\text { acentuadas }\end{array}$ & $\begin{array}{l}\text { Retração intercostal } \\
\text { leve ou ausente }\end{array}$ \\
\hline Sibilos & $\begin{array}{c}\text { Ausentes com } \\
\text { MV } \downarrow / \text { localizados ou difusos }\end{array}$ & Localizados ou difusos & $\begin{array}{l}\text { Ausentes C/ MV } \\
\text { normal/localizados } \\
\text { ou difusos }\end{array}$ \\
\hline F Respiratória (irm)** & Aumentada & Aumentada & $\begin{array}{l}\text { Normal ou } \\
\text { aumentada }\end{array}$ \\
\hline $\mathrm{FC}(\mathrm{bpm}) * * *$ & $>140$ ou bradicardia & $>110$ & $\leq 110$ \\
\hline $\begin{array}{l}\text { Pico de fluxo expiratório } \\
\text { (\% melhor ou previsto) }\end{array}$ & $<30 \%$ & $30-50 \%$ & $>50 \%$ \\
\hline $\mathrm{SaO}_{2}$ (ar ambiente) & $<90 \%$ & $91-95 \%$ & $>95 \%$ \\
\hline $\mathrm{PaO}_{2}$ (ar ambiente) & $<60 \mathrm{mmHg}$ & Ao redor de $60 \mathrm{mmHg}$ & Normal \\
\hline $\mathrm{PaCO}_{2}$ (ar ambiente) & $>45 \mathrm{mmHg}$ & $<40 \mathrm{mmHg}$ & $<40 \mathrm{mmHg}$ \\
\hline
\end{tabular}

\begin{tabular}{|c|c|}
\hline \multicolumn{2}{|r|}{$\begin{array}{c}\text { Q UADRO } 6 \\
\text { Indicações de exames }\end{array}$} \\
\hline Gasometria & Sinais de gravidade, PFE $<30 \%$ após tratamento ou $\mathrm{SaO}_{2} \leq 93 \%$ \\
\hline Radiografia do tórax & $\begin{array}{l}\text { Possibilidade de pneumotórax, pneumonia ou necessidade de internação por crise } \\
\text { grave }\end{array}$ \\
\hline Hemograma & $\begin{array}{l}\text { Suspeita de infecção. N eutrófilos aumentam quatro horas após o uso de corticoste- } \\
\text { róides sistêmicos }\end{array}$ \\
\hline Eletrólitos & $\begin{array}{l}\text { Coexistência com doenças cardiovasculares, uso de diuréticos ou de altas doses de } \\
\beta_{2} \text {-agonistas, especialmente se associados a xantinas e corticosteróides }\end{array}$ \\
\hline
\end{tabular}




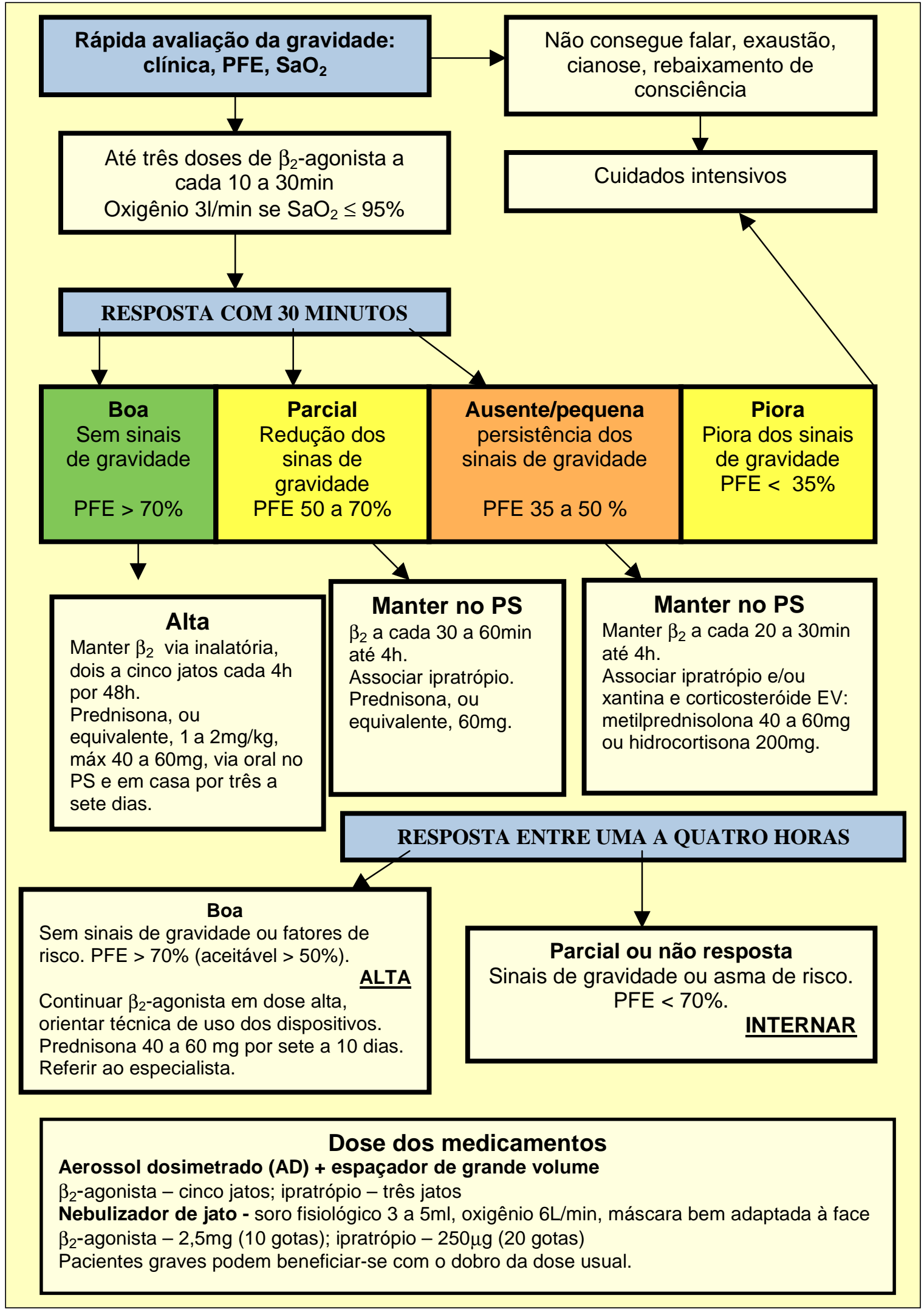

Figura 1 - Algoritmo de tratamento da crise de asma do adulto no pronto-socorro 


\section{Avaliação inicial: FR, FC e PFE, uso de musculatura acessória, dispnéia, grau de alerta, cor, $\mathrm{SatO}_{2}$.}

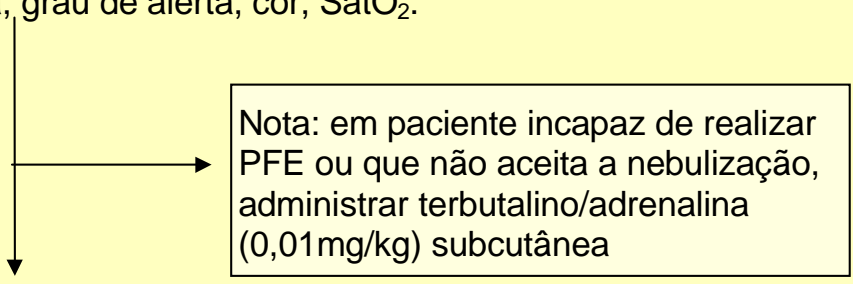

$\mathrm{O}_{2}$ para $\mathrm{SatO}_{2} \leq 95 \%$. Nebulização com $\beta_{2}$-agonista $\left(0,1 \mathrm{mg} / \mathrm{kg} / \mathrm{dose}\right.$ - máx. $5 \mathrm{mg} / \mathrm{dose}$ - fluxo mínimo de $\mathrm{O}_{2}$ de $6 \mathrm{~L} / \mathrm{min})$ ou spray/inaladores de pó (200-300 mcg/dose) a cada 20 minutos, até uma hora (três doses). Em crises graves associar brometo de ipratrópio $(250-500 \mathrm{mcg} / \mathrm{dose}-20-40$ gotas). Se PFE > 90\% após o tratamento inicial, doses adicionais não são necessárias. Iniciar corticosteróides se o paciente é córtico-dependente ou não responder ao tratamento com nebulização.

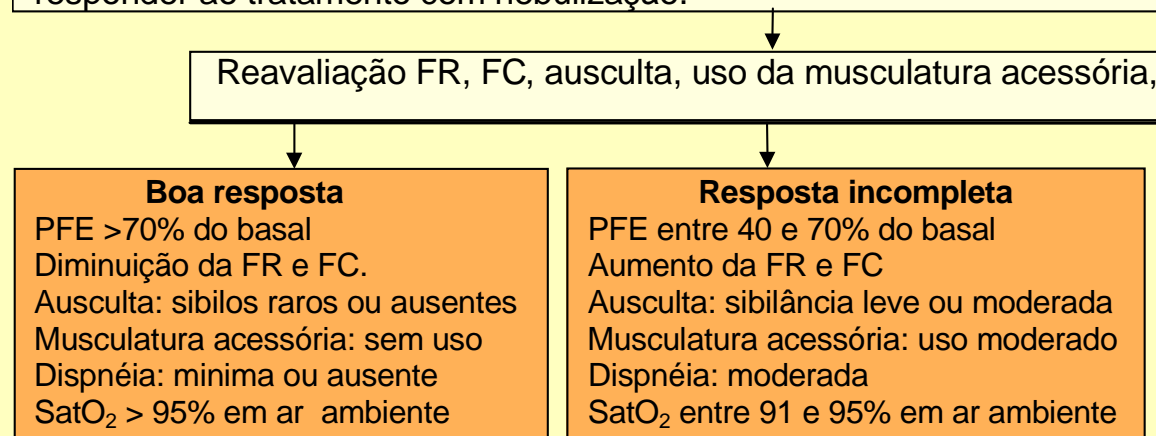

Dispnéia: minima ou ausente

$\mathrm{SatO}_{2}>95 \%$ em ar ambiente

\section{Aumentar intervalos da nebulizações para cada duas horas}

\section{Estável
$\mathrm{PFE}>70 \%$ do basal
$\mathrm{SatO} \mathrm{O}_{2}>95 \%$ e outros
parâmetros
melhorando}

\section{Alta domiciliar com orientação,} $\beta_{2}$ nebulização/spray/inaladores de pó (considerar corticóides orais) e plano de acompanhamento

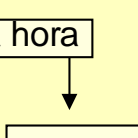

PFE $<70 \%$ do basal $\mathrm{SatO}_{2}<95 \%$, outros parâmetros sem melhora
Manter/adicionar prednisona oral ou similar (1-2 mg/kg -máx 40mg) e continuar nebulização cada $20 \mathrm{~min} \operatorname{com} \beta_{2}(0,15 \mathrm{mg} / \mathrm{kg})$

PFE < $40 \%$ do basal
Aumento da FR e FC
Ausculta: diminuição
entrada de ar
Musculatura acessória:
uso importante
Dispnéia : intensa
$\mathrm{SatO}_{2}<91 \%$ em ar ambiente
PFE $<40 \%$ do basa $\mathrm{SatO}_{2}<91 \%$ em ar ambiente

Reavaliar a gravidade em uma hora

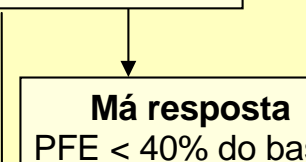

PFE $<40 \%$ do basal $\mathrm{SatO}_{2}<91 \%$ e outros parâmetros sem melhora outros parâmetros melhorando

\section{Boa resposta} PFE $>70 \%$ do basal $\mathrm{SatO}_{2}>95 \%$ e melhorando
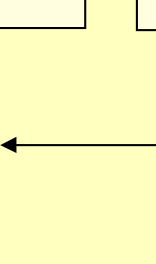

Resposta Incompleta PFE 40 a $70 \%$ do basal $\mathrm{SatO}_{2}$ entre 91 e $95 \%$ e melhorando outros parâmetros

Continuar tratamento.

Considerar internação hospitalar se não houver melhora 
jato/minuto. Em crianças as doses são de 2-3 jatos a cada 20 minutos. 0 limite de dose deverá ser estabelecido observando-se aumento exagerado da FC $>140 \mathrm{bpm}$, tremor grosseiro e arritmias ${ }^{(56)}$.

- Na crise grave, a primeira escolha deve ser a associação dos anticolinérgicos aos $\beta_{2}$-agonistas ${ }^{(57-60)}$.

- Aminofilina não tem indicação como tratamento inicial. Em pacientes muito graves, hospitalizados, ela poderá ser considerada como tratamento adjuvante ${ }^{(22,61,62)}$.

- Corticosteróides reduzem a inflamação, aceleram a recuperação e diminuem o risco de crise fatal. Os pacientes atendidos na emergência devem usar corticosteróides sistêmicos precocemente, embora estudos tenham demonstrado que sua ação não é imediata e que os corticosteróides inalatórios podem ter efeito protetor mais rápido ${ }^{(43,63-65)}$.

- O uso de corticosteróide por via oral ou endovenosa tem efeito equivalente. Os pacientes com alta da emergência que necessitam de corticosteróides devem ser dispensados com prescrição de corticosteróide oral por cinco a 10 dias (para adultos, 40-60 mg/dia e, para crianças, $1-2 \mathrm{mg} / \mathrm{kg} / \mathrm{dia}$, máximo $40 \mathrm{mg} / \mathrm{dia})^{(65-68)}$.

\subsection{Avaliação da resposta ao tratamento}

A resposta ao tratamento inicial (entre $30-60 \mathrm{~min}$ ) e a reclassificação do paciente quanto à gravidade representam os critérios mais úteis para determinar o prognóstico com respeito à admissão e alta e à necessidade de medicação posterior.

\subsection{ERROS E DEFICIÊNCIAS MAIS COMUNS DURANTE O TRA- TAMENTO NO PRONTO-SOCORRO}

- História e exame físicos inadequados

- Falta de medidas funcionais para avaliação da gravidade e da resposta ao tratamento

- Não identificação de asma de risco

- Uso de aminofilina como tratamento principal

- Subdoses de $\beta_{2}$-agonistas ou grande intervalo entre as doses

- Dose insuficiente ou demora na administração de corticosteróides

- Na alta:

- Liberação precoce do pronto-socorro

- Falta de orientação da técnica de uso dos aerossóis

- Não orientar tratamento a longo prazo

- Não orientar sobre retorno e sinais de piora

o Não prescrever prednisona ou equivalente

o Não encaminhar aos serviços de maior complexidade ou ao especialista os casos necessários.

\section{ReferênCIAS}

1. Nelson HS. Beta-adrenergic bronchodilator. N Engl J Med 1995;333: 499-506.
2. Riso JA. Broncodilatadores beta adrenérgicos de longa duração. Segurança, eficácia e indicações. J Pneumol 1997;23:93-100.

3. National Institutes of Health and National Heart, Lung and Blood Institute. Expert panel report 2: Practical guide for diagnosis and management of asthma. NIH Publ 97, 4051, 1997.

4. Sociedade Brasileira de Pneumologia e Tisiologia. II Consenso brasileiro no manejo da asma. J Pneumol 1998;4:173-276.

5. Estelle F, Simons R. A comparison of beclometasone, salmeterol, and placebo in children with asthma. N Engl J Med 1997;337:1659-65.

6. Kavuru M, Melamed J, Gross G, Laforce C, House K, Prillaman B, et al. Salmeterol and fluticasone propionate combined in a new powder inhalation device for the treatment of asthma: a randomized, doubleblind, placebo-controlled trial. J Allergy Clin Immunol 2000;105(6Pt 1):1108-16.

7. Lazarus SC, Boushey HA, Fahy JV, Chinchilli VM, Lemanske RF, Sorkness $C A$, et al. Long-action beta2-agonist monotherapy vs continued therapy with inhaled corticosteroids in patients with persistent asthma: a randomized controlled trial. JAMA 2001;285:2583-93.

8. Lemanske RF, Sorkness CA, Mauger EA, Lazarus SC, Boushey HA, Fahy JV. Inhaled corticosteroid reduction and elimination in patients with persistent asthma receiving salmeterol: a randomized controlled trial. J AMA 2001;285:2594-603.

9. Orsida BE, Ward C, Li X, Bish R, Wilson JW, Thien F, et al. Effect of a long-acting beta2-agonist over three months on airway wall-vascular remodeling in asthma. Am J Respir Crit Care Med 2001;164:117-21.

10. Korosec M, Novak RD, Skowronski M, McFadden ER J r. Salmeterol does not compromise the bronchodilator response to albuterol during acute episodes of asthma. Am J Med 1999;107:209-13.

11. Tattersfield AE, Lafdahl CG, Postma DS, Eivindson A, Schreurs AG, Rasidakis A, Ekstrom T. Comparison of formoterol and terbutaline for as-needed treatment of asthma: a randomised trial. Lancet 2001;357: 257-61.

12. Chervinsy P, Goldberg P, Galant S, Arledge T. Long term cardiovascular safety of salmeterol powder pharmacotherapy in adolescent and adult patients with chronic persistent asthma: a randomized clinical trial. Chest 1999;115:642-8.

13. Ramsdell JW, Klinger NM, Ekholm BP, Colice GL. Safety of long-term treatment with HFA albuterol. Chest 2002;115:945-51.

14. Greening AP, Ind PW, Northfield M, Shaw G. Added salmeterol versus higher-dose corticosteroid in asthma patients with symptoms on existing inhaled corticosteroid. Lancet 1994;344:219-24.

15. Kips J C, O'Connor BJ , Inman MD, Svensson K, Pauwels RA, O'Byrne $P M$. A long-term study of the antiinflammatory effect of low-dose budesonide plus formoterol versus high-dose budesonide in asthma. Am J Resp Crit Care Med 2000;161: 996-1001.

16. Nelson H. Advair: combination treatment with fluticasone propionate/ salmeterol in the treatment of asthma. J Allergy Clin Immunol 2001; 107:397-416

17. Pauwels RA, Lofdahl CG, Postma DS, Tattersfield AE, O'Byrne P, Barnes PJ, et al. Effect of inhaled formoterol and budesonide on exacerbations of asthma. N Engl J Med 1997;337:1405-11.

18. Gross N. Ipratropium bromide. N Engl J Med 1988;319:486-94.

19. Rodrigo J, Rodrigo C. First-line therapy for adults patients with acute asthma receiving a multiple-dose protocol of ipratropium bromide plus albuterol in the emergency department. Am J Resp Crit Care Med 2000; 161:1862-8.

20. Weinberg $M, H$ endeles L. Drug therapy: theophylline in asthma. $N$ Engl J Med 1996;334:1380-8.

21. Reed CE, Offord KP, Nelson HS, Li JT, Tinkelman DG. Aerosol beclomethasone dipropionate spray compared with theophylline as primary treatment for chronic mild-to-moderate asthma. J Allergy Clin Immunol 1998;101:14-23.

22. Rodrigo C, Rodrigo G. Treatment of acute asthma lack of therapeutic benefit and increase of the toxicity from aminophylline given in addition to high doses of salbutamol delivered by metered-dose inhaler with a spacer. Chest 1994;106:1071-6. 
23. Evans DJ, Taylor MB, Zetterstrom O, Chung KF, O'Connor BJ, Barnes PJ. A comparison of low-dose inhaled budesonide plus theophylline and high-dose inhaled budesonide for moderate asthma. N Engl J Med 1997;337: 1412-8.

24. Wiegand L, Mende CN, Zaidel MS, Zwillich CW, Petrocella VJ, Yancey SW, et al. Salmeterol vs theophylline: sleep and efficacy outcomes in patients with nocturnal asthma. Chest 1999;115:1525-32.

25. Barnes PJ, Pedersen S. Efficacy and safety of inhaled corticosteroids in asthma. Am J Resp Crit Care Med 1993;148:s1-s26.

26. Suissa S, Ernst P. Inhaled corticosteroid: impact on asthma morbidity and mortality. J Allergy Clin Immunol 2001;107:937-44.

27. Toogood JH, White FA, Baskerville J C, Fraher LJ , J ennings B. Comparison of the antiasthmatic oropharingeal and systemic glucocorticoid effects of budesonide administered through pressurized aerosol plus spacer or the Turbuhaler dry powder inhaler. J Allergy Clin Immunol 1997;99:186-93.

28. Osterman K, Carlholm M, Ekelund J, Kiviloog J, Nikander K, Nilholm $\mathrm{L}$, et al. Effect of 1 year daily treatment with 400 microg budesonide in newly diagnosed asthmatics. Eur J Respir Dis 1997;10:2210-5.

29. Weiner $P$, Weiner $M$, Azgad Y. Long term clinical comparison of single versus twice daily administration of inhaled budesonide in moderate asthma. Thorax 1995;50:1270-3.

30. Lipworth RJ . Systemic adverse effects of inhaled corticosteroid therapy: a systematic review and meta-analysis. Arch Intern Med 1999;10: 941-55.

31. Barnes PJ, Grunstein MM, Leff AR. Asthma. Philadelphia: LippincottRaven, 1997;2183p.

32. Cumming RG, Mitchell DJ, Leedsee SR. Use de inhaled corticosteroids and risk of cataracts. N Engl J Med 1997;337:8-140.

33. Benedictis FM, Teper A, Green RJ, Boner AL, Williams L, Medley $H$. Effects of 2 inhaled corticosteroids on growth. Arch Pediatr Adolesc Med 2001;155:1248-54.

34. Turktas I, Ozkaya O, Bostanci I, Bideci A, Cinaz P. Safety of inhaled corticosteroid therapy in young children with asthma. Ann Allergy Asthma Immunol 2001;86:649-54.

35. Andersson F, Kjellman M, Forsberg G, Moller C, Arheden L. Comparison of the cost-effectiveness of budesonide and sodium cromoglycate in the management of childhood asthma in everyday clinical practice. Ann Allergy Asthma Immunol 2001;86:537-44.

36. Rossi J A. Nedocromil sódico. J Pneumol 1995;21:295-300.

37. Dempsey OJ, Kennedy G, Lipworth BJ. Comparative efficacy and antiinflammatory profile of one daily therapy with leukotriene antagonist or low-dose inhaled corticosteroid in patients with mild persistent asthma. J Allergy Clin Immunol 2002;109:68-74.

38. Salvi SS, Krishna MT, Sampsom AP. The antiinflammatory effects of leukotrine-modifying drugs and their use in asthma. Chest 2001;119: 1533-46.

39. Vidal C, Fernandez-O vide E, Pineiro J, Nunez R, Gonzalez-Quintela A. Comparison of montelukast versus budesonide in the treatment of exercise-induced bronchoconstriction. Ann Allergy Asthma Immunol 2001:86:655-8.

40. Virchou J C, Prasse A, Naya I. Zafirlukast improves control in patients receiving high-dose inhaled corticosteroids. Am J Respir Crit Care Med 2000;162:578-85

41. Wechsler M, Finn D, Westlake R, Barker A, Haranath SP, Pauwels RA, et al. Churg-Strauss syndrome in patients receiving montelukast as treatment for asthma. Chest 2000;117:708-13.

42. Agertoft $L$, Pedersen $S$. A randomized, double-blind dose reduction study to compare the minimal effective dose of budesonide turbuhaler and fluticosone propionate diskhaler. J Allergy Clin Immunol 1997;99: 773-80.

43. Rowe BH, Spooner CH, Ducharme FM, Bretzlaff JA, Bota GW. Corticosteroids for prevening relapse following acute exacerbation asthma. Cochrane Database Syst Rev 2000;2(CD 000195).

44. Ariyananda PL, Agnew JE, Clarke SW. Aerosol delivery systems for bronchial asthma. Posgrad Med J 1996;72:151-6.
45. O'Callaghan C, Barry PW. The science of nebulized drug delivery. Thorax 1997;52(Suppl 2):s31-s44.

46. Pedersen S. Inhalers and nebulizers: which to choose and why. Respir Med 1996;90:69-77.

47. Pereira LFF. Como administrar drogas por via inalatória. J Pneumol 1998;24:133-43.

48. Siafkas NM. Inhalation therapy. Where do we go from now? Eur Respir Rev 1994;4:64-109.

49. Tashkin DP. 21st century management of upper respiratory allergic diseases: a focus on allergy and asthma. New devices for asthma. J Allergy Clin Immunol 1998;101:409-16.

50. Ellman MS, Viscoli CM, Sears MR, Taylor DR, Beckett WS, Horwitz RI. A new index of prognostic severity for chronic asthma. Chest 1997; 112:582-90.

51. Morris N, Abramson MJ, Rosier MJ, Strasser RP. Assessment of the severity of asthma in a family practice. J Asthma 1996;33:425-39.

52. Wahlgren DR, Hovell MF, Matt GE, Meltzer SB, Zakarian J M, Meltzer EO. Toward a simplified measure of asthma severity for applied research. J Asthma 1997;34:291-303.

53. Brenner B, Konh MS. The acute asthmatic patient in the ED: to admit or discharge. Am J Emerg Med 1998;16:69-75.

54. Gluckman TJ, Corbridge T. Management of respiratory failure in patients with asthma. Curr Opin Pulm Med 2000;6:79-85.

55. Peat JK, Woolcock AJ, Cullen K. Rate of decline of lung function in subjects with asthma. Eur J Respir Dis 1987;70:171.

56. Idris AH, McDermott MF, Raucci JC, Morrabel A, McGorray S, Hendeles $\mathrm{L}$. Emergency department treatment of severe asthma. Metereddose inhaler plus holding chamber is equivalent in effectiveness to nebulizer. Chest 1993;103:665-72.

57. Lin RY, Pesola GR, Bakalchuk L, Morgan JP, Heyl GT, Freyberg CW, et al. Superiority of ipratropium plus albuterol over albuterol alone in the emergency department management of adult asthma: a randomized clinical trial. Ann Emerg Med 1998;31:208-13.

58. Qureshi F, Pestian J, Davis P, Zaritsky A. Effect of nebulized ipratropium on the hospitalization rates of children with asthma. N Engl J Med 1998;339:1030-5.

59. Qureshi F, Zaritsky A, Lakkis H. Efficacy of nebulized ipratropium in severely asthmatic children. Ann Emerg Med 1997;29:205-11.

60. Stoodley RG, Aaron SD, Dales RE. The role of ipratropium bromide in the emergency management of acute asthma exacerbation: a metaanalysis of randomized clinical trials. Ann Emerg Med 1999;34:8-18.

61. Hart SP. Should aminophylline be abandoned in the treatment of acute asthma in adults? QJ M 2000;93:761-5.

62. Yung $M$, South $M$. Randomized controlled trial of aminophyline for severe acute asthma. Arch Dis Child 1998;79:405-10.

63. Lin RY, Pesola GR, Bacalchuck, Heyl GT, Dow AM, Tenebaum C, et al. Rapid improvement of peak flow in asthmatic patients treated with parenteral methylprednisolone in the emergency department: a randomized controlled study. Am Emerg Med 1999; 33:487-94.

64. Rowe BH, Spooner C, Ducharme FM, Bretzlaff JA, Bota GW. Early emergency department treatment of acute asthma with systemic corticosteroids. Cochrane Database Syst Rev 2000;CD002178.

65. Volovitz $B$, Bentur L, Finkelstein $Y$, Mansour Y. Effectiveness and safety of inhaled corticosteroids in controlling acute asthma attacks in children who were treated in the emergency department: a controlled comparative study with oral prednisolone. J Allergy Clin Immunol 1998; 102:605-9.

66. Afilalo M, Guttman A, Dankoff J, Tselioss C, Stern E, Wolkove N, et al. Efficacy of inhaled steroids (beclomethasone diproprionate) for treatment of mild to moderately severe asthma in the emergency department: a randomized clinical trial. Ann Emerg Med 1999;33:304-9.

67. FitzGerald J M, Shragge D, Haddon J, J ennings B, Lee J, Bai T, et al. A randomized controlled trial of high dose inhaled budesonide versus oral prednisone in patients discharged from the emergency department following an acute asthma exacerbation. Can Respir J 2000;7:61-7.

68. Nana A, Youngchaiyud P, Charoenratanakul S, Boe J, Lofdahl CG, Selroos $\mathrm{O}$, et al. High dose inhaled budesonide may substitute for oral therapy after an acute asthma attack. J Asthma 1998;35:647-55. 